\title{
Lubrication and Fatigue Life Evaluation of High-Speed Cylindrical Roller Bearing under Misalignment
}

\author{
Hongyu Duan, ${ }^{1,2}$ Jiawei Song, ${ }^{3}$ and Zhijian Wang $\mathbb{D}^{3}$ \\ ${ }^{1}$ School of Mechatronics Engineering and Automation, Shanghai University, Shanghai 200072, China \\ ${ }^{2}$ Shanghai Bearing Technology Research Institute, Shanghai 200072, China \\ ${ }^{3}$ School of Mechanical Engineering and Rail Transit, Changzhou University, Changzhou, China
}

Correspondence should be addressed to Zhijian Wang; wzj10721180@163.com

Received 15 July 2020; Revised 27 August 2020; Accepted 19 September 2020; Published 5 October 2020

Academic Editor: Suzanne M. Shontz

Copyright (c) 2020 Hongyu Duan et al. This is an open access article distributed under the Creative Commons Attribution License, which permits unrestricted use, distribution, and reproduction in any medium, provided the original work is properly cited.

\begin{abstract}
This paper developed a coupled model, incorporating the quasistatic model, fatigue life model, and mixed lubrication model, to investigate the effect of misalignment angle on high-speed cylindrical roller bearings. The model is verified by comparing with the published literature results. Then, a parametric analysis is carried out. The results show that as the misalignment increases, the load distribution is basically unchanged, but the fatigue life of the roller bearing decreases due to the variation of contact pressure, and the skewing moment of single roller contact pair increases. Furthermore, the optimal design of roller profile needs to consider the effect of lubrication in order to improve the fatigue life of roller bearings. In general, the optimal crown drop is too small according to the design from the slicing technique.
\end{abstract}

\section{Introduction}

Cylindrical roller bearings are often used in high-speed engine applications. In these applications, cylindrical roller bearings are very sensitive to the misalignment of the ring which can lead to the reduction of fatigue life and the cage instability. However, in some demanding applications, the misalignment can be easily caused by improper assembly or shaft bending under load. Thus, it is necessary to study the performance of high-speed cylindrical roller bearings under misalignment and put forward some corresponding optimal designs.

An early study about the effect of misalignment on fatigue life of cylindrical roller bearings was investigated by Harris [1]. He used the slicing technique to determine the load per length along the roller. Liu [2] further extended Harris's method by considering the effect of centrifugal forces. Ye et al. [3] combined the quasidynamic model and FEM to calculate load distribution and contact pressure under misalignment. Warda and Chudzik [4] applied the quasistatic model to obtain the load distribution and then evaluated the fatigue life under misalignment by calculating the subsurface stresses. Tong et al. [5] established a four-degree-of-freedom quasistatic model to evaluate the potential error sources on fatigue life and used the 3D contact model to provide the contact pressure distributions in rolling elements. However, it should be pointed out that the effect of lubrication on performance of high-speed cylindrical roller bearings under misalignment is overlooked in most of works above. Experiments by Tallian [6] and Liu et al. [7] have demonstrated that the film thickness has a significant effect on the fatigue life of rolling bearings. Kushwaha et al. [8] and Liu et al. $[9,10]$ investigated the lubrication mechanism of tilting roller pairs and found that the symmetry of film pressure and thickness along the roller is destroyed. In load-carrying end of roller, the film thickness sharply decreases which may lead to the early failure of the roller bearing. In addition, asymmetrical film pressure would produce skewing moments about the roller center and cause the skewing of roller. Recently, Shi and Wang [11] coupled the quasidynamic model with the elasto-hydrodynamic lubrication model to evaluate the lubrication performance of cylindrical roller bearings. 
The object of this work is to investigate the effect of misalignment on the lubrication and life of high-speed cylindrical roller bearings. The coupled quasistatic and mixed lubrication model will be applied to evaluate the load distribution, lubrication condition, fatigue life, and skewing moments of the single roller contact pair under a misaligned operating condition. Some optimum geometric conditions for reliable operation of high-speed cylindrical roller bearings under misalignment will be established.

\section{Model}

In this part, the coupled model, the quasistatic model, and mixed lubrication model will be described. First, the quasistatic model is used to calculate the load distribution among the rolling elements and the magnitude of misalignment of the individual roller. Then, these results are applied to the mixed lubrication model which considers the effect of roughness and elastoplastic deformation of asperities. By the mixed lubrication model, the film thickness distribution along the roller axial directions is calculated. Last, the fatigue life considering lubrication will be evaluated by the fatigue life model. The solution process of the couple model is illustrated in Figure 1.

2.1. Quasistatic Model of Cylindrical Roller Bearings. In this section, the quasistatic model developed by Liu [2] is adopted to evaluate the bearing load distribution. To commence the analysis, the roller-raceway contact is divided into a number of slices. According to Palmgren [12], the relationships of deformation $\delta$ and load $Q$ can be described as follows:

$$
\delta=3.84 \times 10^{-5} \frac{Q^{0.9}}{L^{0.8}}
$$

where $L$ is the roller effective contact length.

Suppose that the contact is divided into $k$ laminas, each lamina of width $w$, as shown in Figure 2. Let $q=Q / L$; then,

$$
q=\frac{\delta^{1.11}}{1.24 \times 10^{-5}(k w)^{0.11}},
$$

where $q$ is the contact load per unit length.

Figure 3 shows the misalignment of bearing inner ring. From Figure 3, it can be found that the misalignment angle of the inner ring is $\theta . B_{j}$ is the only point on the inner raceway that will maintain the same distance from the fixed outer ring before and after the misalignment. The $x$ coordinate of the point $B_{j}$ is

$$
x_{B j}=-r \tan \left(\frac{1}{2} \theta \cos \psi_{j}\right) .
$$

Due to radial displacement $\delta_{r}$, the radial deformation of the roller at angular location $\psi_{j}$ is

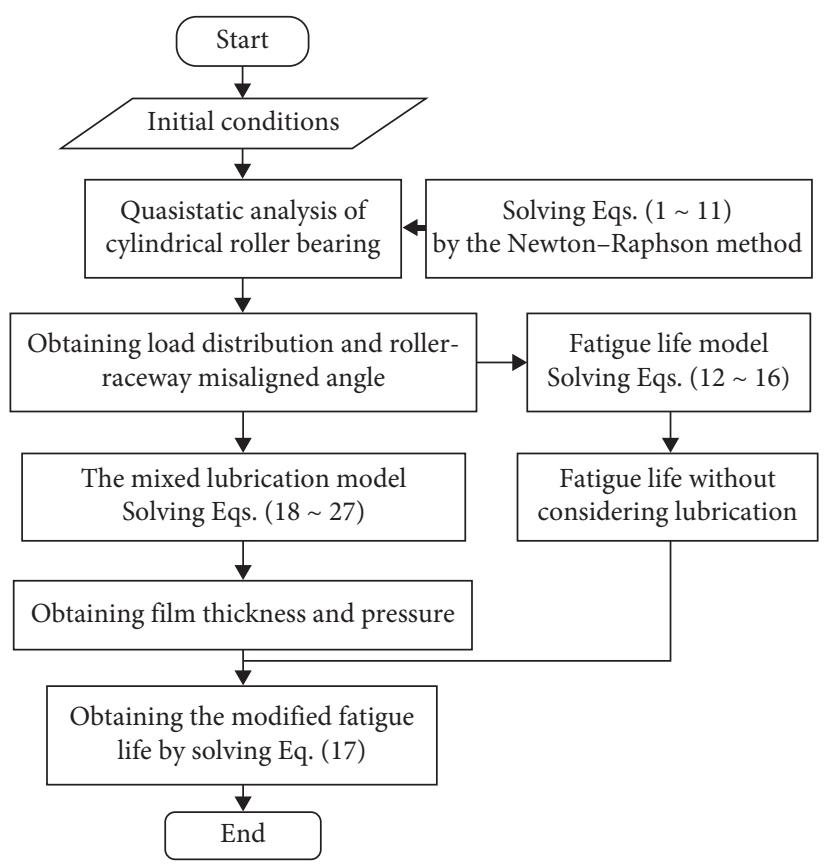

FIGURE 1: Flowchart of numerical solution.

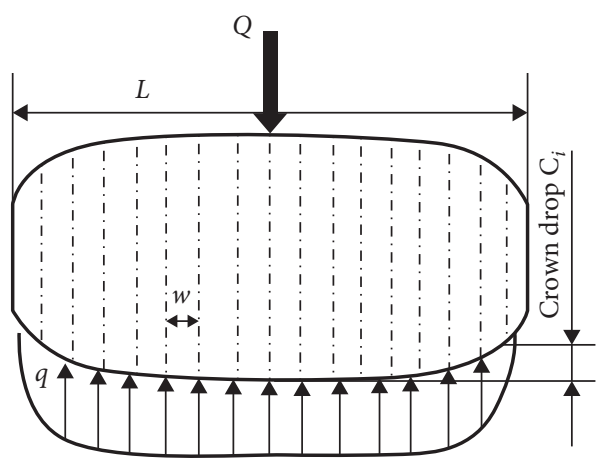

Figure 2: The roller profile.

$$
\begin{aligned}
& \delta_{\psi_{j}}=\delta_{r} \cos \psi_{j}-\frac{P_{d}}{2}, \\
& \delta_{\psi_{j}}^{I}=\delta_{r} \cos \psi_{j}-\frac{P_{d}}{2}-\delta_{\psi_{j}}^{O},
\end{aligned}
$$

where $P_{d}$ is the radial clearance of the bearing.

Designate the roller tilt angle as $\beta_{j}$ at angular location $\psi_{j}$; then, the total contact deformation of segment $i$ of the roller at $\psi_{j}$ is

$$
\delta_{i j}^{o}=\delta_{\psi_{j}}^{O}+\left[x_{i}+r \tan \left(\frac{1}{2} \theta \cos \psi_{j}\right)\right] \tan \left( \pm \beta_{j}\right)-C_{i},
$$

for the outer contact and

$$
\begin{aligned}
\delta_{i j}^{I}= & \delta_{r} \cos \psi_{j}-\frac{P_{d}}{2}-\delta_{\psi_{j}}^{O} \\
& +\left[x_{i}+r \tan \left(\frac{1}{2} \theta \cos \psi_{j}\right)\right] \tan \left(\theta \cos \psi_{j} \mp \beta_{j}\right)-C_{i},
\end{aligned}
$$




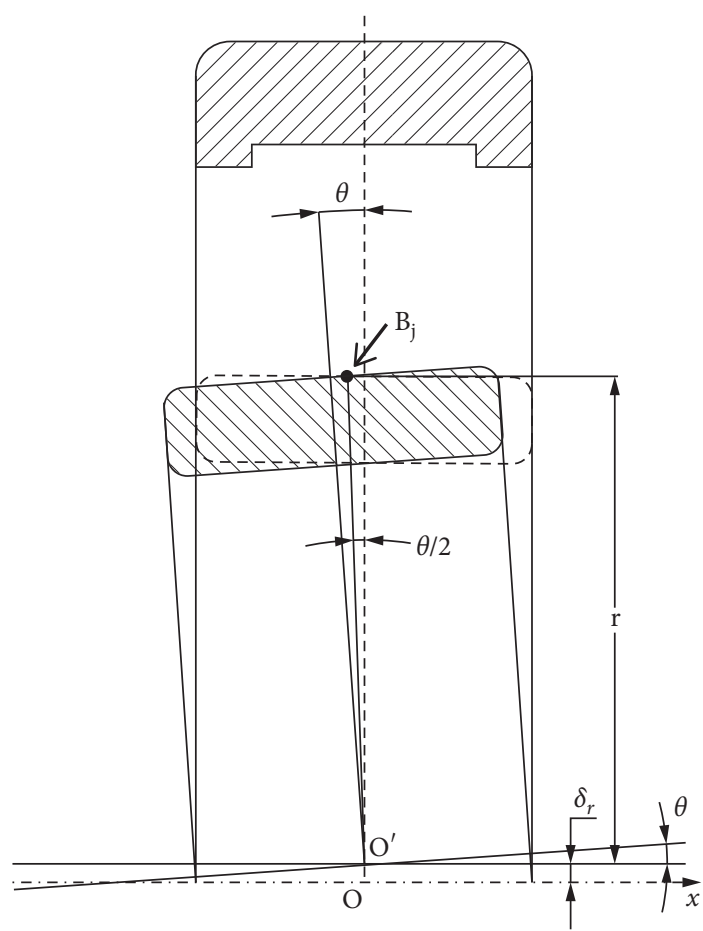

Figure 3: Misalignment of bearing inner ring.

for the inner contact, where $C_{i}$ is crown drop at segment $i$, as shown in Figure 2. In the next analysis, for convenience, the crown drop at $8.5 \%$ of the roller length from the end is used to describe the variation of the roller profile.

Thus, the load intensity on the roller at $\psi_{j}$ can be obtained from equation (2), as follows:

$$
\begin{aligned}
& q_{i j}^{o}=\frac{\left(\delta_{i j}^{o}\right)^{1.11}}{1.24 \times 10^{-5}(k w)^{0.11}}, \\
& q_{i j}^{I}=\frac{\left(\delta_{i j}^{I}\right)^{1.11}}{1.24 \times 10^{-5}(k w)^{0.11}} .
\end{aligned}
$$

The static equilibrium of the roller is

$$
w \sum_{i=1}^{k} q_{i j}^{I}-w \sum_{i=1}^{k} q_{i j}^{o}+F_{c}=0,
$$

where $F_{c}$ is the roller centrifugal force.

The equilibrium equation of the bearing is

$$
F-\sum_{j=1}^{z} w \sum_{i=1}^{k} q_{i j}^{I}=0
$$

where $z$ is the number of rollers.

These nonlinear equations can be solved by the Newton-Raphson iterative technique. The detailed modeling process and numerical solution can be found in reference [2].

\subsection{Cylindrical Roller Bearing Fatigue Life Formulation and} Correction Factor. According to [13, 14], $L_{10}$ fatigue life of a roller-raceway line contact subjected to normal load $Q$ may be estimated by

$$
L_{10}=\left(\frac{Q_{c}}{Q}\right)^{4},
$$

where $L_{10}$ is in millions of revolutions and $Q_{c}$ is the basic dynamic load ratings of inner and outer ring.

$$
Q_{c}=552 \frac{(1 \mp \gamma)^{(29 / 27)}}{(1 \pm \gamma)^{(1 / 4)}} \gamma^{(2 / 9)} D^{(29 / 27)} L^{(7 / 9)} Z^{(-1 / 4)},
$$

where $D$ is the roller diameter, $\gamma=D / d m$, and $d m$ is the pitch diameter of the bearing. The upper signs refer to the inner ring and the lower signs refer to the outer ring.

The basic of load rating of a slice is expressed as follows:

$$
q_{c, i, o}=Q_{c}\left(\frac{1}{k}\right)^{(7 / 9)} \text {. }
$$

The equivalent load of a slice is calculated as

$$
q(i)^{i, o}=\left(\frac{1}{Z} \sum_{j=1}^{z}\left(q_{i j}^{i, o} w\right)^{4}\right)^{(1 / 4)} .
$$

Thus, the basic reference rating life, $L_{10 r}$, is

$$
L_{10 r}=\left\{\sum_{i=1}^{k}\left[\left(\frac{q_{c i}}{q(i)^{i}}\right)^{-4.5}+\left(\frac{q_{c o}}{q(i)^{o}}\right)^{-4.5}\right]\right\}^{(-8 / 9)} .
$$

Zaretsky et al. [15] recommended the mean curve to estimate the effect of lubrication on bearing fatigue life, as shown in Figure 4. By regression analysis, a fourth-order polynomial expression can be used to describe this mean curve, as follows:

$$
\begin{aligned}
L= & -\left(1.6249+3.03381 \Lambda-0.78723 \Lambda^{2}\right. \\
& \left.+0.08918 \Lambda^{3}-0.00365 \Lambda^{4}\right) L_{10 r},
\end{aligned}
$$

where $\Lambda$ is the film parameter, $\Lambda=\left(h_{\min } /\left(\sqrt{\sigma_{1}^{2}+\sigma_{2}^{2}}\right)\right), h_{\text {min }}$ is the minimum film thickness, which can be obtained from the following mixed lubrication model, $\sigma_{1}$ is the root mean square (rms) of the raceway surface, and $\sigma_{2}$ is rms roughness of the roller surface.

It should be noted that when the film parameter is less than 0.6 , the predicted error may increase. In this situation, the life of bearings mainly depends on the properties of the boundary films. Thus, the fatigue life may be more reliable when the film parameter is larger than 0.6 in the current model.

2.3. Mixed Lubrication Model of Single Roller Contact Pairs. The modified Reynolds equation derived by Patir and Cheng $[16]$ is

$$
\begin{aligned}
& \frac{\partial}{\partial x}\left(\phi_{x} \frac{\rho h^{3}}{12 \eta} \frac{\partial p_{h}}{\partial x}\right)+\frac{\partial}{\partial y}\left(\phi_{y} \frac{\rho h^{3}}{12 \eta} \frac{\partial p_{h}}{\partial y}\right) \\
& =\frac{\partial \rho h_{T}}{\partial x}\left(\frac{u_{1}+u_{2}}{2}\right)+\frac{u_{1}-u_{2}}{2} \sigma \frac{\partial\left(\rho \phi_{s}\right)}{\partial x},
\end{aligned}
$$

where $h$ is the nominal film thickness, $\eta$ is the lubricant viscosity, $\rho$ is the lubrication density, $h_{T}$ is the average gap 


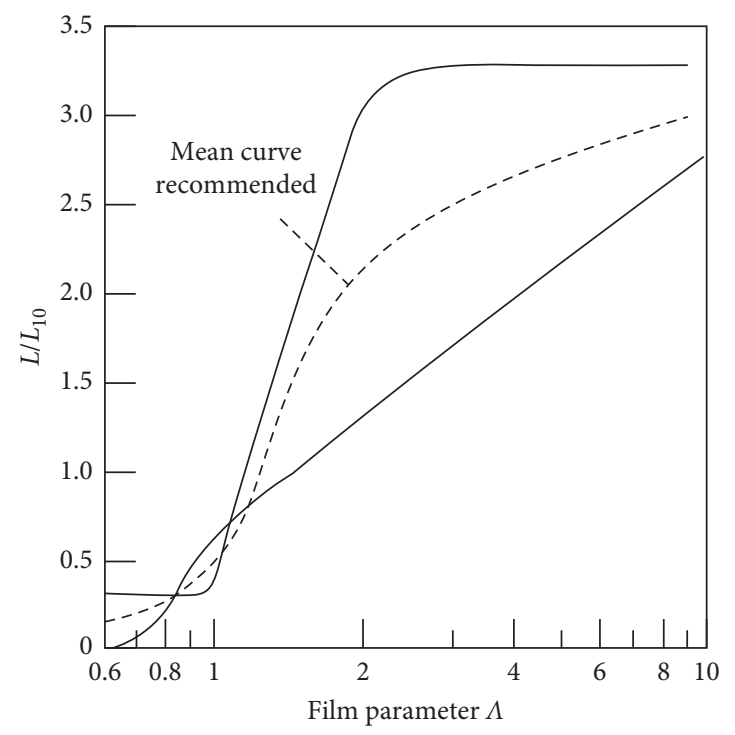

FIgURE 4: The effect of film parameter on bearing fatigue life.

between the two surfaces, $u_{1}$ and $u_{2}$ are the velocities $(x$ direction) of the two surfaces, $\phi_{x}$ and $\phi_{y}$ are the pressure flow factor in $x$ and $y$ direction, $x$ and $y$ are the coordinates along and perpendicular to the rolling direction, respectively (see Figure 1), $\phi_{s}$ is the shear flow factor, and $\sigma$ is the standard deviation of combined surface roughness $\left(\sigma=\sqrt{\sigma_{1}^{2}+\sigma_{2}^{2}}\right)$.

For an isotropic surface with Gaussian distribution of the surfaces heights, the pressure and the shear flow factors are given by Patir and Cheng [16].

$$
\begin{aligned}
& \phi_{x}=\phi_{y}=1-0.9 e^{-0.56(h / \sigma)}, \\
& \phi_{s}=\left(\frac{\sigma_{1}}{\sigma}\right)^{2} \Phi_{s}\left(\frac{h}{\sigma}, 1\right)-\left(\frac{\sigma_{2}}{\sigma}\right)^{2} \Phi_{s}\left(\frac{h}{\sigma}, 1\right),
\end{aligned}
$$

where $\Phi_{s}$ is a positive function of $(h / \sigma)$ and the surface pattern parameter of a given surface.

For the Gaussian distribution of the surfaces heights, $h_{T}$ can be written as $[16$

$$
h_{T}=0.5 h\left[1+\operatorname{erf}\left(\frac{h}{\sqrt{2} \sigma}\right)\right]+\left(\frac{\sigma}{\sqrt{2 \pi}}\right) \exp \left(\frac{-h^{2}}{2 \sigma^{2}}\right) .
$$

In equation (16), viscosity and density are calculated by the Roelands equation [17] and Dowson and Higginson equation [18], respectively.

$$
\begin{aligned}
& \eta=\eta_{0} \exp \left\{A_{1}\left[-1+\left(1+A_{2} p_{h}\right)^{Z_{0}}\right]\right\}, \\
& \rho=\rho_{0}\left[1+\frac{C_{1} p_{h}}{1+C_{2} p_{h}}\right],
\end{aligned}
$$

where $\eta_{0}$ and $\rho_{0}$ are the viscosity and density at atmospheric pressure and temperature, $z_{0}$ is the viscosity-pressure coefficient, $A_{1}=\ln \eta_{0}+9.67, A_{2}=5.1 \times 10^{-9} \mathrm{~Pa}^{-1}, C_{1}=0.6 \times$ $10^{-9} \mathrm{~Pa}^{-1}$, and $C_{2}=1.7 \times 10^{-9} \mathrm{~Pa}^{-1}$.

The lubricant film thickness $h$ in equation (16) can be written as

$$
\begin{aligned}
h(x, y)= & h_{00}+s(x, y) \\
& +\frac{2}{\pi E^{\prime}} \iint_{\Omega} \frac{p(s, q)}{\sqrt{(x-s)^{2}+(y-q)^{2}}} \mathrm{~d} s \mathrm{~d} q+y \tan \gamma,
\end{aligned}
$$

where $h_{00}$ is the normal approach of two surfaces, $s(x, y)$ is the initial gap depending on the roller and raceway prolife, $E^{\prime}$ is the equivalent modulus of elasticity, $\Omega$ is the calculating area, and $\gamma$ is the relative misalignment angle, which can be calculated as follows:

$$
\gamma=\theta \cos \psi-\beta
$$

for the inner ring and

$$
\gamma=\beta
$$

for the outer ring.

The elastic-plastic microcontact model developed by Zhao et al. [19] is applied. The expression of asperity contact pressure is given in equation (23), which incorporates the transition from elastic to fully plastic deformation. 


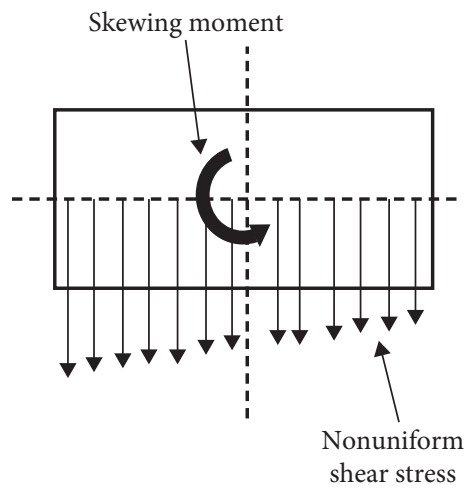

FIGURE 5: Skewing moment.

$$
\begin{aligned}
p_{a}= & \frac{2}{3} E^{\prime} n \beta^{0.5} \sigma^{1.5}\left(\frac{\sigma}{\sigma_{s}}\right) \frac{1}{\sqrt{2 \pi}} \int_{h^{*}-y_{s}^{*}}^{h^{*}-y_{s}^{*}+\omega_{1}^{*}} w^{* 1.5} e^{-0.5\left(\left(\sigma / \sigma_{s}\right) z^{*}\right)^{2}} \mathrm{~d} z^{*} \\
& +2 \pi \times h d \times n \beta \sigma \frac{1}{\sqrt{2 \pi}}\left(\frac{\sigma}{\sigma_{s}}\right) \int_{h^{*}-y_{s}^{*}+\omega_{2}^{*}}^{\infty} w^{*} e^{-0.5\left(\left(\sigma / \sigma_{s}\right) z^{*}\right)^{2}} \mathrm{~d} z^{*} \\
& +\pi \times h d \times n \beta \sigma \frac{1}{\sqrt{2 \pi}}\left(\frac{\sigma}{\sigma_{s}}\right) \int_{h^{*}-y_{s}^{*}+\omega_{1}^{*}}^{h^{*}-y_{s}^{*}+\omega_{s}^{*}} w^{*} e^{-0.5\left(\left(\sigma / \sigma_{s}\right) z^{*}\right)^{2}} \times\left[1-0.6 \frac{\ln \omega_{2}^{*}-\ln \omega^{*}}{\ln \omega_{2}^{*}-\ln \omega_{1}^{*}}\right] \times\left[1-2\left(\frac{\omega^{*}-\omega_{1}^{*}}{\omega_{2}^{*}-\omega_{1}^{*}}\right)^{3}+3\left(\frac{\omega^{*}-\omega_{1}^{*}}{\omega_{2}^{*}-\omega_{1}^{*}}\right)^{2}\right] \mathrm{d} z^{*},
\end{aligned}
$$

where $\omega^{*}=z^{*}-h^{*}+y_{s}^{*}$. In equation (23), the starred variables are normalized by $\sigma, n$ is the asperity density, $h d$ is the softer material's hardness, $\beta$ is the asperity radius, $y_{s}$ is the distance between the mean line of the surface and the mean line of its summits, and $\sigma_{s}$ is the standard deviation of the surface summits.

The total load $w$ is given by

$$
\iint_{\Omega} p_{h}(x, y) \mathrm{d} x \mathrm{~d} y+\iint_{\Omega} p_{a}(x, y) \mathrm{d} x \mathrm{~d} y=w,
$$

where $p_{h}$ is calculated by equation (16), and the load carried by the asperities $w_{a}$ is calculated by

$$
w_{a}=\iint_{\Omega} p_{a}(x, y) \mathrm{d} x \mathrm{~d} y .
$$

In order to evaluate skewing moment more accurately, the friction coefficient in each contact grid needs to be calculated. Based on Johnson et al.'s load-sharing concept [20], the total applied load in each grid is supported by hydrodynamic force and asperity interacting force. Therefore, the average friction coefficient $\mu$ in each grid can be expressed as follows:

$$
\mu(x, y)=\frac{\tau_{f}(x, y)+\mu_{a} p_{a}(x, y)}{p_{a}(x, y)+p_{h}(x, y)},
$$

where $\tau_{f}$ is the film shear stress and $\mu_{a}$ is the friction coefficient of asperity contact. Generally, the value of $\mu_{a}$ depends on the properties of boundary film; here assuming the physical absorbed film exists, its value is assumed to be 0.08 . [21].

$$
\tau_{f}=\tau_{0} \arcsin h\left(\frac{\eta \dot{\gamma}}{\tau_{0}}\right),
$$

where $\tau_{0}$ is the Eyring stress of the lubricant.

The skewing moment is caused by nonuniform shear stress, as shown in Figure 5, and can be calculated by

$$
M_{s}=\iint_{\Omega} y \mu(x, y)\left(p_{a}(x, y)+p_{h}(x, y)\right) \mathrm{d} x \mathrm{~d} y .
$$

Once these three models are connected, the coupled model outputs the following useful variables to evaluate the tribological performance of high-speed cylindrical roller bearings under misalignment:

(a) The inner/outer ring contact load in different misalignment angles

(b) The film pressure/thickness profile in different misalignment angles

(c) The fatigue life of high-speed cylindrical roller bearing under misalignment

(d) The skewing moment of single roller contact pairs under misalignment

\section{Results and Discussion}

3.1. Verification. The coupled model mainly includes two parts: quasistatic model and mixed lubrication model under misaligned operating conditions. The results of the quasistatic model are compared with the results from reference [2]. The input parameters are identical. The results are shown in Figure 6. It can be found that the present results are very close to the reference results. Furthermore, the mixed lubrication model has been verified and used in reference [22]. Thus, the coupled model can be applied in the next analysis. 


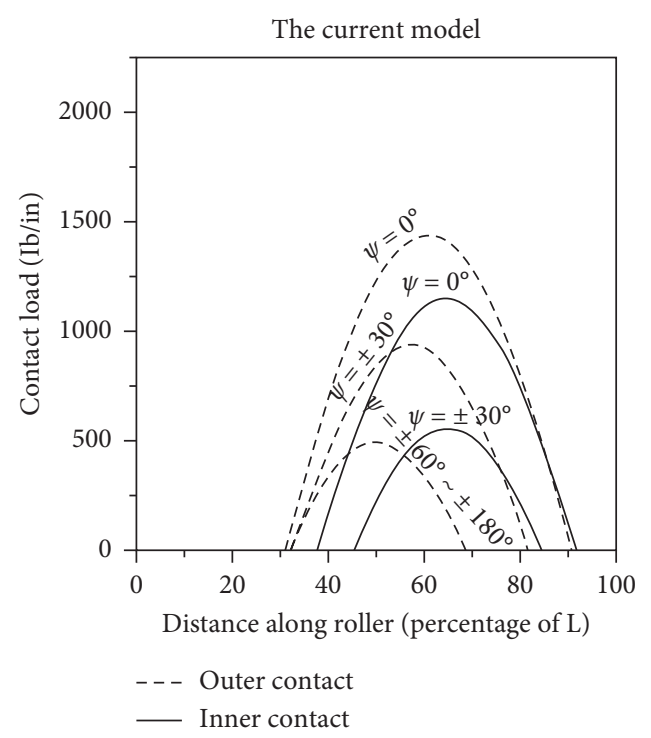

(a)

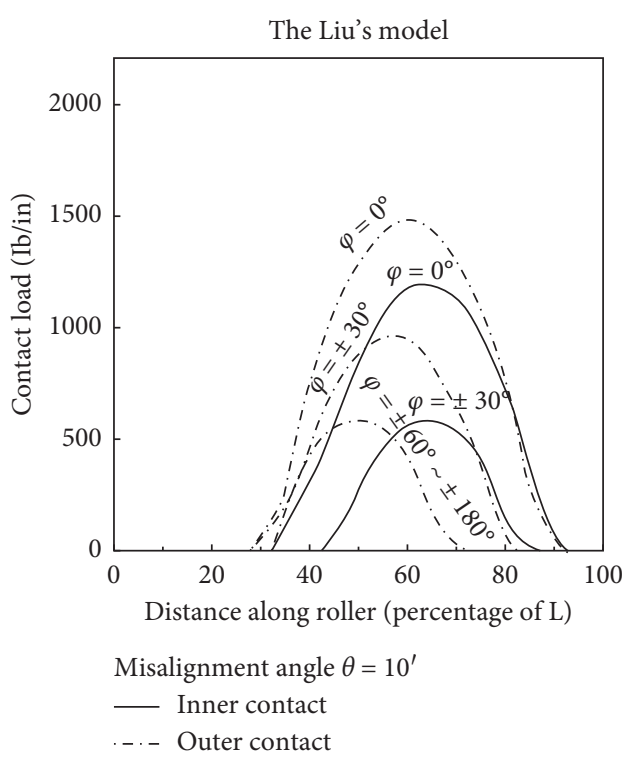

(b)

Figure 6: Verification of the quasistatic model.

3.2. Parametric Analysis. A high-speed cylindrical roller bearing used in a helicopter turbine engine is analyzed. Its geometrical and material parameters are listed in Table 1. The lubricant is a kind of synthetic aviation turbine engine oil with the kinematic viscosity of $3.765 \mathrm{~mm}^{2} / \mathrm{s}$ at $100^{\circ} \mathrm{C}$. The viscosity-pressure coefficient is $12.8 \mathrm{GPa}^{-1}$. The bearings operate at an applying load of $6780 \mathrm{~N}$ and a rotational speed of $10000 \mathrm{rpm}$. The logarithmic profile roller is applied and can be described as follows:

$$
C(y)=2 \frac{1-v^{2}}{\pi E} \frac{Q_{s}}{L}\left(\ln \frac{1}{1-(2 y / L)^{2}}\right),
$$

where $Q_{s}$ is the designed load; the crown drop of the roller can be changed by the variation of $Q_{s}$. In the current analysis, $Q_{s}$ is equal to $15000 \mathrm{~N}$.

\subsubsection{The Effect of Misalignment on Load Distribution and} Fatigue Life. Figures 6-8 show the influence of misalignment on load distribution and fatigue life; here the effect of lubrication on fatigue life is not confided. From Figure 7, it can be found that the outer raceway contact load distribution is basically unchanged with increasing misalignment angle. For the outer raceway contact, the lowest contact load is equal to centrifugal force. It should be noted that though the load distribution is unchanged, the contact loads along the single roller axial direction change. Take the heaviest loaded roller for example, as shown in Figure 8; it can be seen that the roller gives uniform contact load in the aligned bearings. When the ring misaligns, asymmetric contact load occurs. And the maximum contact load considerably increases with the increase of misalignment angle which would result in the reduction of fatigue life, as shown in Figure 9. When the misalignment angle reaches 10 minutes, the fatigue life drops to $31.9 \%$ compared with aligned
Table 1: The related data of cylindrical roller bearing.

\begin{tabular}{lc}
\hline Inner raceway diameter, $\mathrm{mm}$ & 51.92 \\
Outer raceway diameter, mm & 79.95 \\
Roller diameter, mm & 13.99 \\
Roller effective length, mm & 14.68 \\
Number of rollers & 12 \\
Roughness of raceway, $\mu \mathrm{m}$ & 0.05 \\
Roughness of roller, $\mu \mathrm{m}$ & 0.06 \\
Elastic moduli of roller and rings, $\mathrm{MPa}$ & 206000 \\
Poisson's ratios of roller and rings & 0.3 \\
Density of rollers, $\mathrm{kg} / \mathrm{m}^{3}$ & 7850 \\
\hline
\end{tabular}

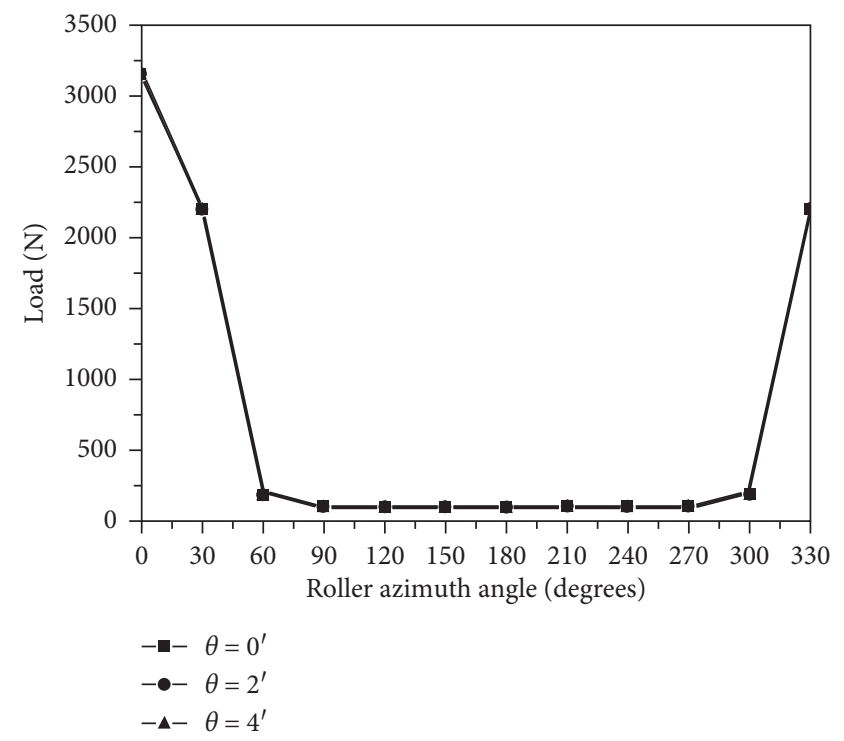

FIgURE 7: Outer raceway contact load versus roller location. 


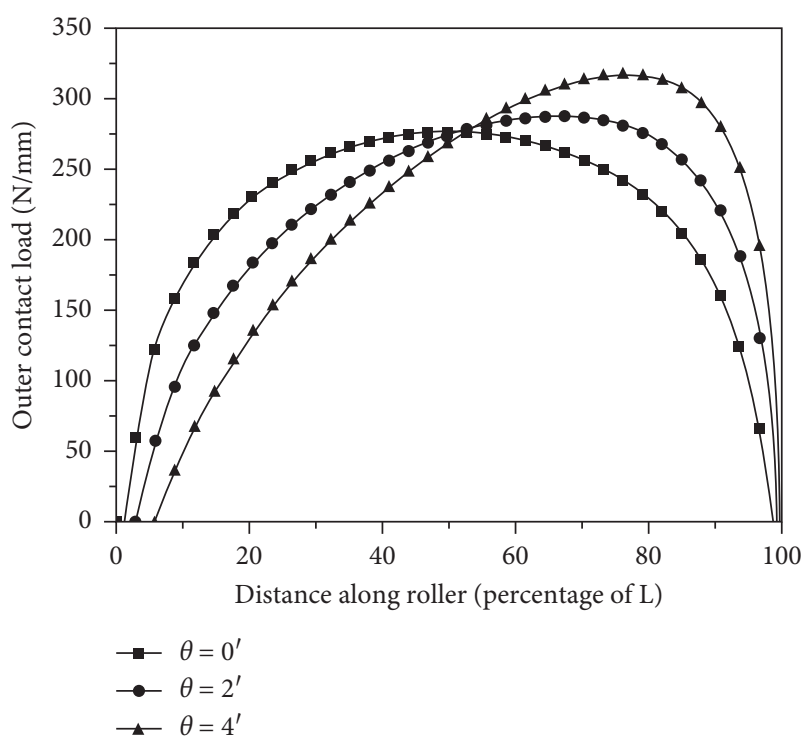

FIGURE 8: Contact load variation along roller axial direction.

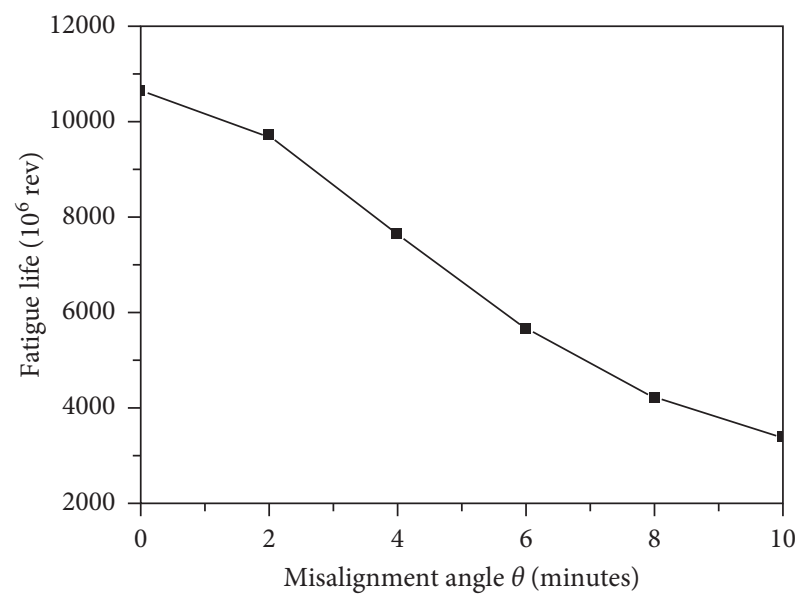

Figure 9: The effect of misalignment on fatigue life (without considering the effect of lubrication).

bearing which indicates that even very small misalignment angle can cause a substantial reduction in the fatigue life.

\subsubsection{The Effect of Misalignment on Lubrication Performance} and Fatigue Life. In this section, the results from the heaviest loaded roller are analyzed. The basic operating parameters are listed in Table 2. Figure 10 shows the variation of contact pressure and film thickness along the roller axial direction with increasing misalignment angle. It can be seen that in the loadcarrying end of the roller, the contact pressure increases and the film thickness decreases with increasing misalignment angle. When the misalignment angle is above 4 minutes, the minimum film thickness sharply reduces. Comparing the contact pressure and film thickness between the inner contact and outer contact, it can be found that the inner contact pressure is higher and the film thickness is thinner which indicates that the lubrication and contact condition in the inner contact is more severe.
TABLE 2: The basic operating parameters in EHL analysis.

\begin{tabular}{|c|c|c|c|c|c|}
\hline $\begin{array}{l}\text { Misalignment } \\
\text { angle }\end{array}$ & & $\begin{array}{l}\text { Load } \\
(\mathrm{N})\end{array}$ & $\begin{array}{c}\text { Roller relative } \\
\text { misalignment } \\
\text { angle } \gamma\left({ }^{\prime}\right)\end{array}$ & $\begin{array}{l}\text { The } \\
\text { equivalent } \\
\text { radius } \\
(\mathrm{mm})\end{array}$ & $\begin{array}{l}\text { Velocity } \\
(\mathrm{m} / \mathrm{s})\end{array}$ \\
\hline \multirow{2}{*}{$0^{\prime}$} & $\begin{array}{l}\text { Inner } \\
\text { contact }\end{array}$ & 3073 & 0 & 5.512 & 16.48 \\
\hline & $\begin{array}{c}\text { Outer } \\
\text { contact }\end{array}$ & 3158 & 0 & 8.482 & 16.48 \\
\hline \multirow{2}{*}{$2^{\prime}$} & $\begin{array}{l}\text { Inner } \\
\text { contact }\end{array}$ & 3064 & 1.01 & 5.512 & 16.48 \\
\hline & $\begin{array}{c}\text { Outer } \\
\text { contact }\end{array}$ & 3149 & 0.99 & 8.482 & 16.48 \\
\hline \multirow{2}{*}{$4^{\prime}$} & $\begin{array}{c}\text { Inner } \\
\text { contact }\end{array}$ & 3034 & 2.01 & 5.512 & 16.48 \\
\hline & $\begin{array}{c}\text { Outer } \\
\text { contact }\end{array}$ & 3133 & 1.99 & 8.482 & 16.48 \\
\hline
\end{tabular}

Figure 11 shows the effect of lubrication on film parameter and asperity-carrying load; it can be seen that as the misalignment angle increases, the film parameter decreases. When the misalignment angle is above 8 minutes, the minimum film thickness is equal to zero which indicates that the entire load in some grid is fully carried by asperities. In addition, the asperitycarrying load increases with increasing misalignment angle. In general, in the current analysis, the percentage of load carried by asperities is relatively small due to the high entering speed and the high precision contact surfaces. However, when the misalignment angle is above 6 minutes, the trend of increasing changes and sharply increases. Thus, the results in Figure 10 suggest that the misalignment angle should be lower than 6 minutes in order to operate reliably.

Figure 12 shows the effect of lubrication on fatigue life. It can be found that when the misalignment angle is less than 4 minutes, the predicted fatigue life in lubrication conditions is longer than that without considering the effect of lubrication. In these cases, the film parameter is larger than 1.0. If the misalignment angle further increases, the fatigue life in lubrication conditions would sharply decrease. When the misalignment angle is larger than 6 minutes, the predicted fatigue life is equal to zero since the evaluated film parameter is equal to zero. However, in this situation, the rolling bearing may still operate over a period of time which depends on the properties of boundary films. It is hard to predict the fatigue life accurately for very low film parameter. Thus, if the predicted fatigue life is equal to zero, it means that the lubrication condition is the most severe, and some improvement measures should be taken.

Figure 13 shows the effect of lubrication on skewing moment. It can be found that as the misalignment angle increases, the skewing moment increases which can cause the skewing angle to increase for a full complement cylindrical roller bearing. Liu et al. [23] investigated the effect of skewing angle on lubrication performance and found that as the skewing angle increases, the minimum film thickness decreases. Thus, the increase of skewing moment may lead to the reduction of bearing fatigue life. In addition, if the skewing angle is controlled by the small clearances between roller and cage pocket or roller and ring flange, it also can cause a series of problems such as the wear on roller ends or cage instability. 


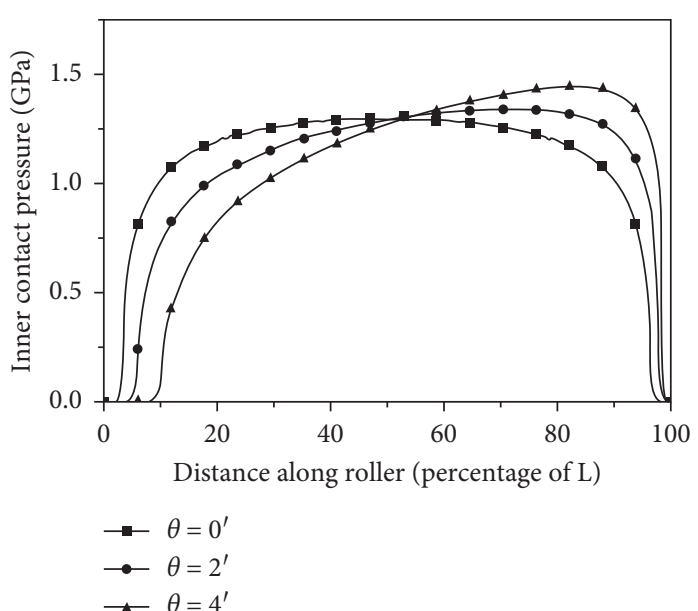

(a)

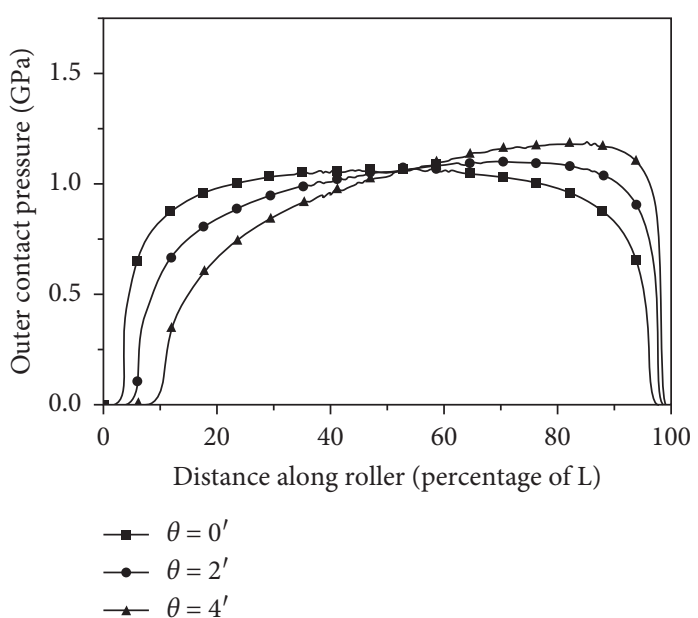

(c)

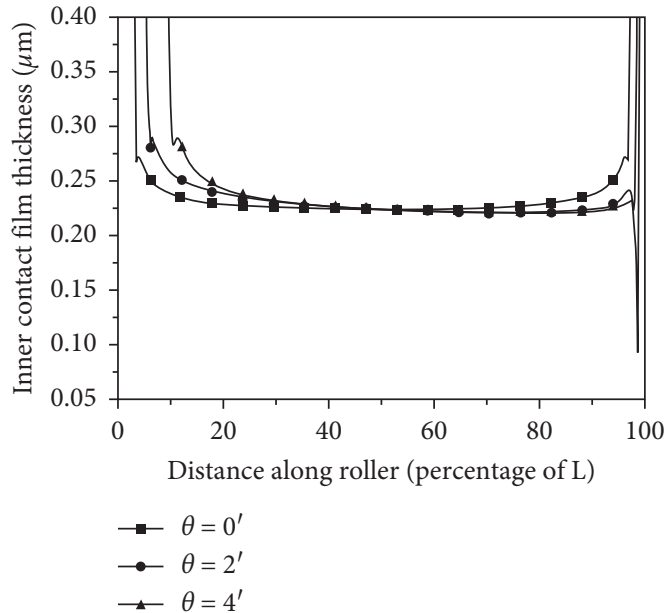

(b)

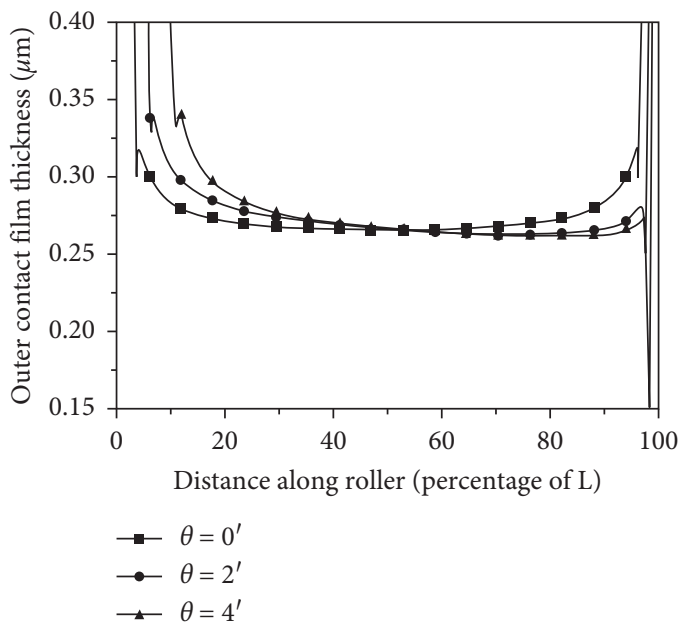

(d)

Figure 10: The effect of misalignment on the contact pressure and film thickness.

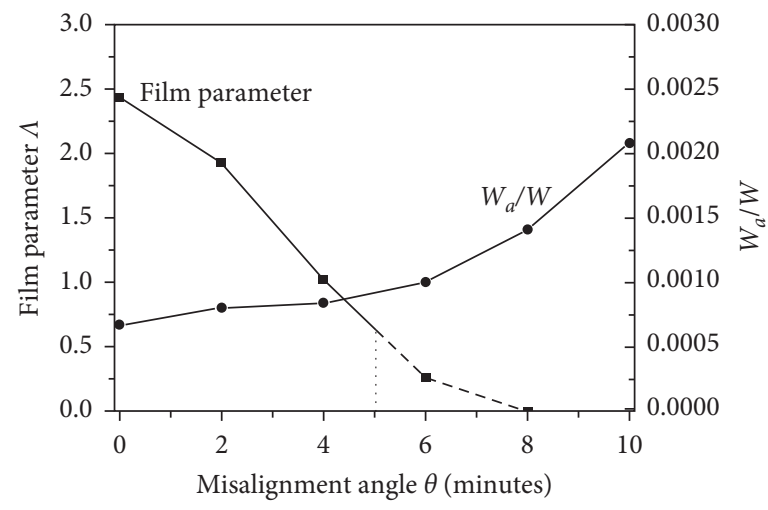

FIgURE 11: The effect of lubrication on film parameter and asperity-carrying load.

3.3. The Optimal Design of Roller Profile. From the above analysis, it can be found that in large misaligned operating condition, the fatigue life would be greatly reduced due to lubrication failure. In order to improve this situation, roller profile is an effective method. Lots of theoretical and experimental works $[24,25]$ have investigated the effect of roller profile on fatigue life. In the current analysis, based on the lubrication numerical simulation, the optimal 


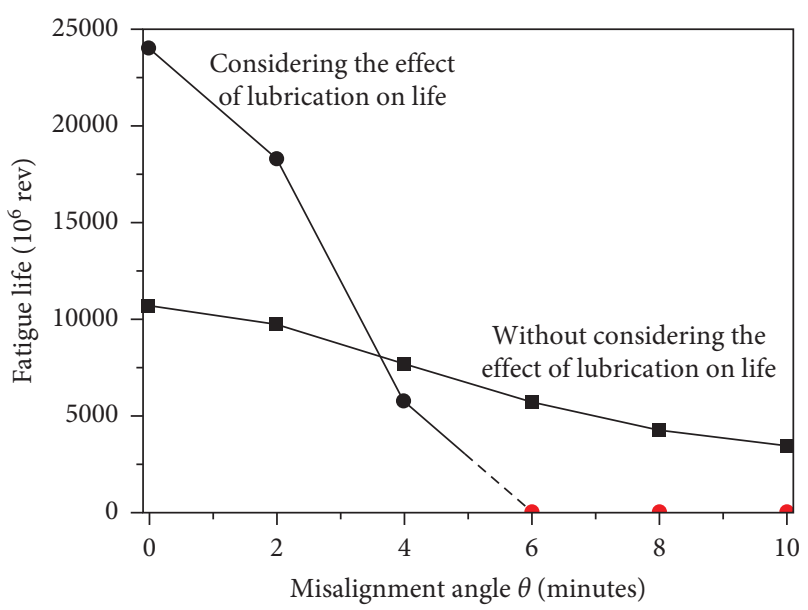

Figure 12: The effect of lubrication on fatigue life.

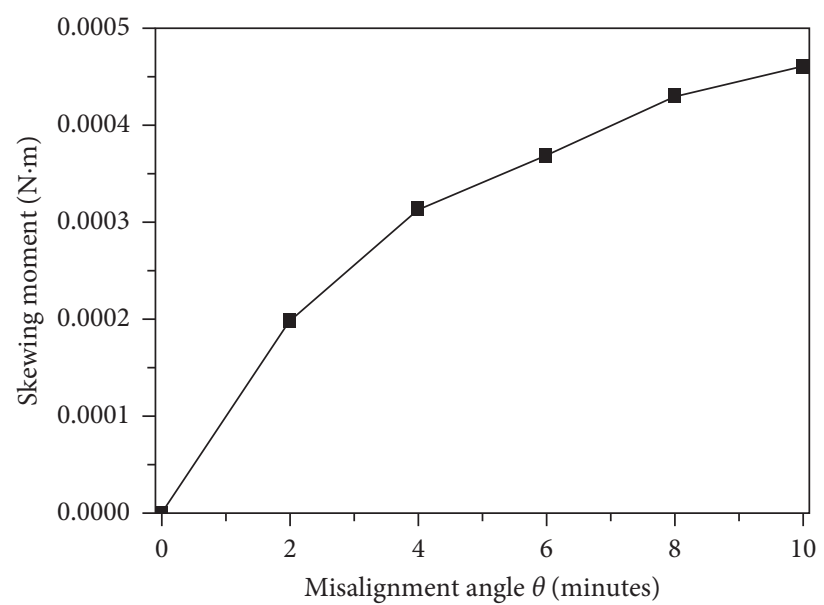

FIgURE 13: The effect of misalignment on skewing moment.

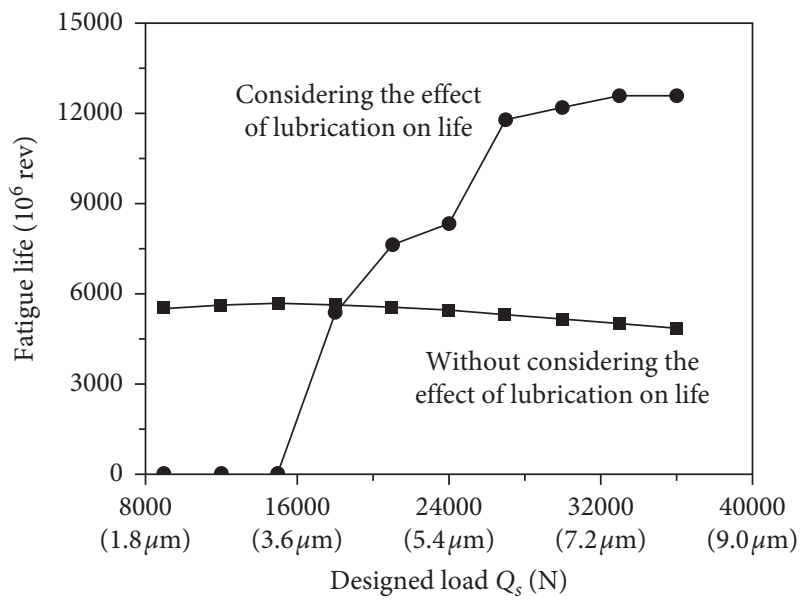

Figure 14: The effect of crown drop on fatigue life.

roller profile under misaligned operating conditions is designed. Figure 14 shows the effect of crown drop on fatigue life under 6 minutes misalignment angle. It should be noted that as the designed load increases, the crown drop increases. Thus, it can be found that if the influence of lubrication on life is not considered, the optimal 
designed load in current operating conditions is equal to $15000 \mathrm{~N}$. However, it can cause lubrication failure in this situation. The crown drop needs to be further increased to improve lubrication performance. When the influence of lubrication is considered, the fatigue life increases with the increase of crown drop. If the designed load is between $18000 \mathrm{~N}$ and $27000 \mathrm{~N}$, the fatigue life significantly increases. After that, the fatigue life slightly increases. Then, the fatigue life may decrease due to high contact pressure in the middle of the roller. Furthermore, though high film parameter can improve fatigue life, it can also increase friction loss. In current operating conditions, the optimal design load is equal to $27000 \mathrm{~N}$. Therefore, the results in Figure 13 hint that the design of reasonable roller profile should consider the effect of lubrication.

\section{Conclusions}

In this study, a coupled model for evaluating the lubrication condition and fatigue life of high-speed cylindrical roller bearings under misaligned operating conditions was established. This model incorporates the quasistatic model, fatigue life model, and mixed lubrication model. A parametric analysis was carried out to investigate the effect of misalignment on load distribution, fatigue life, and skewing moment. Some conclusions are drawn as

(1) The misalignment of rings has little influence on load distribution. However, it can greatly reduce fatigue life.

(2) Lubrication plays an important role in operating the roller bearing reliably. For the misaligned operating conditions, the minimum film thickness occurs in the load-carrying end of the roller. As the misalignment angle increases, the minimum film thickness decreases. In current analysis, when the misalignment angle is larger than 6 minutes, the minimum film thickness is equal to zero.

(3) The optimal design of roller profile needs to consider the influence of lubrication. If the effect of lubrication is not considered, the designed crown drop may be too small.

(4) As the misalignment angle increases, the skewing moment of single roller contact pair increases.

\section{Data Availability}

All data generated or analyzed during this study are included in this article. The code used during the current study is available from the corresponding author on reasonable request.

\section{Conflicts of Interest}

The authors declare that they have no potential conflicts of interest with respect to the research, authorship, and publication of this article.

\section{Acknowledgments}

This study was financially supported by the Scientific Research Foundation of Changzhou University.

\section{References}

[1] T. A. Harris, "The effect of misalignment on the fatigue life of cylindrical roller bearings having crowned rolling members," Journal of Lubrication Technology, vol. 91, no. 2, pp. 294-300, 1969.

[2] J. Y. Liu, "The effect of misalignment on the life of high speed cylindrical roller bearings," Journal of Lubrication Technology, vol. 93, no. 1, pp. 60-68, 1971.

[3] Z. Ye, L. Wang, L. Gu, and C. Zhang, "Effects of tilted misalignment on loading characteristics of cylindrical roller bearings," Mechanism and Machine Theory, vol. 69, no. 6, pp. 153-167, 2013.

[4] B. Warda and A. Chudzik, "Effect of ring misalignment on the fatigue life of the radial cylindrical roller bearing," International Journal of Mechanical Sciences, vol. 111-112, pp. 1-11, 2016.

[5] V.-C. Tong, S.-W. Kwon, and S.-W. Hong, "Fatigue life of cylindrical roller bearings," Proceedings of the Institution of Mechanical Engineers, Part J: Journal of Engineering Tribology, vol. 231, no. 5, pp. 623-636, 2017.

[6] T. E. Tallian, "Rolling bearing life modifying factors for film thickness, surface roughness, and friction," Journal of $\mathrm{Lu}$ brication Technology, vol. 103, no. 4, pp. 509-516, 1981.

[7] J. Y. Liu, T. E. Tallian, and J. I. Mccool, "Dependence of bearing fatigue life on film thickness to surface roughness ratio," A S L E Transactions, vol. 18, no. 2, pp. 144-152, 1975.

[8] M. Kushwaha, H. Rahnejat, and R. Gohar, "Aligned and misaligned contacts of rollers to races in elastohydrodynamic finite line conjunctions," Proceedings of the Institution of Mechanical Engineers, Part C: Journal of Mechanical Engineering Science, vol. 216, no. 11, pp. 1051-1070, 2002.

[9] X.-L. Liu, P. Yang, and P.-R. Yang, "Analysis of the lubricating mechanism for tilting rollers in rolling bearings," Proceedings of the Institution of Mechanical Engineers, Part J: Journal of Engineering Tribology, vol. 225, no. 11, pp. 1059-1070, 2011.

[10] X. Liu and P. Yang, "On the thermal elastohydrodynamic lubrication of tilting roller pairs," Tribology International, vol. 65, no. 3, pp. 346-353, 2013.

[11] X. Shi and L. Q. Wang, "TEHL analysis of aero-engine mainshaft roller bearing based on quasi-dynamics," Journal of Mechanical Engineering, vol. 52, no. 3, pp. 86-92, 2016, in Chinese.

[12] A. Palmgren, Ball and Roller Bearing Engineering, SKF Industries Inc., Philadelphia, PA, USA, 1959.

[13] T. A. Harris and M. N. Kotzalas, Essential Concepts of Bearing Technology, CRC Press, Boca Raton, FL, USA, 5 th edition, 2006.

[14] ISO, "Rolling bearings-methods for calculating the modified reference rating life for universally loaded bearings," ISO, Geneva, Switzerland, ISO/TS 16281:2008, 2008.

[15] E. Zaretsky, T. Harris, E. N. Bamberger et al., Life Adjustment Factors for Ball and Roller Bearings, ASME Design Guide, New York, NY, USA, 1971.

[16] N. Patir and H. S. Cheng, "An average flow model for determining effects of three-dimensional roughness on partial hydrodynamic lubrication," Journal of Lubrication Technology, vol. 100, no. 1, pp. 12-17, 1978. 
[17] C. J. A. Roelands, W. O. Winer, and W. A. Wright, "Correlational aspects of the viscosity-temperature-pressure relationship of lubricating oils (Dr in dissertation at technical university of Delft, 1966)," Journal of Lubrication Technology, vol. 93, no. 1, pp. 209-210, 1971.

[18] D. Dowson and G. R. Higginson, Elasto-Hydrodynamic Lubrication, Elsevier, Amsterdam, Netherlands, 1966.

[19] Y. Zhao, D. M. Maietta, and L. Chang, "An asperity microcontact model incorporating the transition from elastic deformation to fully plastic flow," Journal of Tribology, vol. 122, no. 1, pp. 86-93, 1999.

[20] K. L. Johnson, J. A. Greenwood, and S. Y. Poon, "A simple theory of asperity contact in elastohydro-dynamic lubrication," Wear, vol. 19, no. 1, pp. 91-108, 1972.

[21] K. L. Johnson and J. L. Tevaarwerk, "Shear behaviour of elastohydrodynamic oil films," Proceedings of the Royal Society A. Mathematical Physical \& Engineering Sciences, vol. 356, no. 1685, pp. 215-236, 1977.

[22] Z. Wang, X. Chen, X. Shen, and L. Zhou, "Optimum design of the roller profile based on the elastohydrodynamic lubrication model," Proceedings of the Institution of Mechanical Engineers, Part J: Journal of Engineering Tribology, vol. 233, no. 10, pp. 1594-1604, 2019.

[23] X. Liu, X. Bai, J. Cui, and P. Yang, "Thermal elastohydrodynamic lubrication analysis for tilted and skewed rollers in cylindrical roller bearings," Proceedings of the Institution of Mechanical Engineers, Part J: Journal of Engineering Tribology, vol. 230, no. 4, pp. 428-441, 2016.

[24] J. V. Poplawski, S. M. Peters, and E. V. Zaretsky, "Effect of roller profile on cylindrical roller bearing life prediction-Part I: comparison of bearing life theories," Tribology Transactions, vol. 44, no. 3, pp. 339-350, 2001.

[25] J. V. Poplawski, S. M. Peters, and E. V. Zaretsky, "Effect of roller profile on cylindrical roller bearing life prediction-Part II comparison of roller profiles," Tribology Transactions, vol. 44, no. 3, pp. 417-427, 2001. 